\title{
SMOOTH SOLUTIONS TO THE RELATIVISTIC VLASOV-MAXWELL SYSTEM
}

\author{
CHRISTOPHE PALLARD*
}

\begin{abstract}
We focus on the existence of classical solutions to the relativistic Vlasov-Maxwell system of equations. We discuss an alternative proof of the result by Glassey and Strauss showing that smooth solutions do not develop singularities as long as the momentum support remains bounded.
\end{abstract}

Key words. Kinetic equations, Vlasov-Maxwell system.

AMS subject classifications. 82D10, 35Q75, 76X05

\section{Introduction}

1.1. The relativistic Vlasov-Maxwell system. It is a kinetic mean-field model for a collisionless plasma, that is, a gas of charged particles which is sufficiently hot and dilute to consider collisional effects as negligible. The particles are thus supposed to interact only through electromagnetic forces. For a discussion of the physical meaning of these assumptions, see [5] section 5.1 and references therein. We assume that the plasma is made of $n$ different kind of particles (electrons, ions) with masses $m_{\alpha}$ and charges $e_{\alpha}$. As is customary in the framework of statistical physics, the set of all particles of this species is described by a distribution function $f_{\alpha} \equiv f_{\alpha}(t, x, \xi)$ which gives at time $t \geq 0$ a probability density on the phase space $\mathbb{R}_{x}^{3} \times \mathbb{R}_{\xi}^{3}$. The motion of particles is then governed by Vlasov's equation:

$$
\partial_{t} f_{\alpha}+v_{\alpha}(\xi) \cdot \nabla_{x} f_{\alpha}+e_{\alpha}\left(E+\frac{v_{\alpha}}{c} \wedge B\right) \cdot \nabla_{\xi} f_{\alpha}=0
$$

where $c$ is the speed of light and

$$
v_{\alpha}(\xi)=\frac{\xi}{\sqrt{m_{\alpha}^{2}+|\xi|^{2} / c^{2}}}
$$

is the relativistic speed of a particle of species $\alpha$ and momentum $\xi$. Besides, the electric field $E \equiv E(t, x)$ and the magnetic field $B \equiv B(t, x)$ satisfy Maxwell's equations:

$$
\begin{array}{ll}
\partial_{t} E-c \nabla_{x} \wedge B=-j, & \nabla_{x} \cdot E=\rho \\
\partial_{t} B+c \nabla_{x} \wedge E=0, & \nabla_{x} \cdot B=0 .
\end{array}
$$

The density of charge $\rho$ and current $j$ are computed by:

$$
\rho=4 \pi \sum_{\alpha} \int e_{\alpha} f_{\alpha} d \xi, \quad j=4 \pi \sum_{\alpha} \int e_{\alpha} f_{\alpha} v_{\alpha} d \xi .
$$

These equations are supplemented with initial data

$$
\left.f_{\alpha}\right|_{t=0}=f_{\alpha}^{i n},\left.\quad E\right|_{t=0}=E^{i n},\left.\quad B\right|_{\mid t=0}=B^{i n},
$$

which are supposed to satisfy the compatibility condition

$$
\nabla_{x} \cdot E^{i n}=\rho^{i n} \text { and } \nabla_{x} \cdot B^{i n}=0
$$

*Université Paris 7, U. F. R. de Mathématiques, Case 7012, 2 Place Jussieu, 75251 Paris CEDEX 05, France (pallard@math.jussieu.fr). 
and the global neutrality assumption $\int \rho^{i n} d x=0$.

In the sequel we shall consider the case where there is only one species of particles, since the results we discuss here can be easily extended to the case of a plasma made of several species. Also, we set all physical constants equal to one. We obtain:

$$
\partial_{t} f+v(\xi) \cdot \nabla_{x} f+K \cdot \nabla_{\xi} f=0,
$$

with Lorentz force $K=E+v(\xi) \wedge B$ and relativistic velocity $v(\xi)=\frac{\xi}{\sqrt{1+|\xi|^{2}}}$,

$$
\begin{array}{ll}
\partial_{t} E-\nabla_{x} \wedge B=j, & \nabla_{x} \cdot E=\rho \\
\partial_{t} B+\nabla_{x} \wedge E=0, & \nabla_{x} \cdot B=0 .
\end{array}
$$

where $\rho=\int f d \xi$ and $j=\int f v d \xi$. The initial data are given by

$$
\left.f\right|_{t=0}=f^{i n},\left.\quad E\right|_{t=0}=E^{i n},\left.\quad B\right|_{\mid t=0}=B^{i n},
$$

such that $\nabla_{x} \cdot E^{i n}=\rho^{i n}$ and $\nabla_{x} \cdot B=0$. Hereafter, we designate by relativistic VlasovMaxwell system the set of equations (1.6-1.7-1.8).

1.2. A priori estimates. The characteristic curves of the Vlasov equation are defined by equations

$$
\dot{X}(t)=v(\Xi(t)), \quad \dot{\Xi}(t)=E(t, X(t))+v(\Xi(t)) \wedge B(t, X(t)),
$$

and initial conditions $X(0)=x_{0}$ and $\Xi(0)=\xi_{0}$. The distribution function $f$ should be constant along $(X, \Xi)$ :

$$
f(t, X(t), \Xi(t))=f^{i n}\left(x_{0}, \xi_{0}\right),
$$

which leads to the useful estimate

$$
\|f(t, \cdot, \cdot)\|_{L^{\infty}\left(\mathbb{R}^{3} \times \mathbb{R}^{3}\right)}=\left\|f^{i n}\right\|_{L^{\infty}\left(\mathbb{R}^{3} \times \mathbb{R}^{3}\right)} .
$$

Also, since $\nabla_{\xi} \cdot(E+v \wedge B)=0$, we have mass preservation as well:

$$
\|f(, \cdot, \cdot)\|_{L^{1}\left(\mathbb{R}^{3} \times \mathbb{R}^{3}\right)}=\left\|f^{i n}\right\|_{L^{1}\left(\mathbb{R}^{3} \times \mathbb{R}^{3}\right)} .
$$

Another estimate is available through the conservation of the total energy $\mathcal{E}(t)$ defined as

$$
\iint\left(\sqrt{1+|\xi|^{2}}-1\right) f(t, x, \xi) d \xi d x+\frac{1}{2} \int|E(t, x)|^{2}+|B(t, x)|^{2} d x
$$

See proposition 1.6 in [3] for a proof of this claim.

\section{Smooth solutions to RVM}

2.1. Glassey-Strauss' theorem. We recall that the existence of global-intime weak solutions to the RVM system was derived in the late eighties by DiPerna and Lions in [4]. See also [3, 19]. Less is known as far as classical solutions are concerned. The major result obtained in this respect is the one by Glassey and Strauss. In [8], they showed that classical solutions do not develop singularities as 
long as the particles' speed remains bounded away from the speed of light. Let us denote by $R(t)$ the size of the momentum support of the distribution function $f$ :

$$
R(t)=\sup \left\{|\xi|, \exists x \in \mathbb{R}^{3} f(t, x, \xi) \neq 0\right\} .
$$

We are interested here in the following theorem.

TheOREM 2.1. Let $f \in \mathcal{C}^{1}\left([0, T) \times \mathbb{R}^{6}\right)$ and $E, B \in \mathcal{C}^{1}\left([0, T) \times \mathbb{R}^{3}\right)$ be a solution to $(R V M)$ with initial distribution $f^{i n} \in \mathcal{C}_{c}^{1}\left(\mathbb{R}^{6}\right)$ and initial fields $E^{i n}, B^{i n} \in \mathcal{C}_{c}^{2}\left(\mathbb{R}^{3}\right)$ satisfying the compatibility condition. Assume that

$$
r^{*}=\sup _{t \in[0, T)} R(t)<\infty .
$$

Then we have

$$
\|f(t, \cdot, \cdot)\|_{W_{x, \xi}^{1, \infty}}+\|(E, B)(t, \cdot)\|_{W_{x}^{1, \infty}} \leq C\left(r^{*}\right) .
$$

More recently, Klainerman and Staffilani suggested another viewpoint, using Fourier analysis methods and the regularizing effect provided by integrating the fields along the characteristic curves. We refer the interested reader to [14]. In the next sections, we shall discuss at length a proof of theorem 2.1 that can be found in [2].

2.2. The issue of high velocities. The problem with theorem 2.1 is that there is no result so far proving - or disproving - that $R$ does not blow up in finite time for the initial data considered here. Note, however, that such a result is known in the case of the nonrelativistic Vlasov-Poisson system. See [5, 13, 17, 20].

Some efforts have been made to weaken this existence criterion. In [9], Glassey and Strauss have been able to replace it by a condition on moments

$$
M_{\theta}(t, \cdot)=\int f(t, \cdot, \xi)|\xi|^{\theta} d \xi
$$

Namely, they proved that it is enough to assume $M_{1}(t, \cdot) \in L^{\infty}\left(\mathbb{R}^{3}\right)$. In [16], the author showed that $R$ remains bounded as long as $M_{\theta}(t, \cdot) \in L^{p}\left(\mathbb{R}^{3}\right)$ for the range of values $\theta>4 / p$ and $p \geq 6$. This should be compared to the a priori estimate following from the conservation of $(1.13): M_{1}(t, \cdot) \in L^{1}\left(\mathbb{R}^{3}\right)$.

We end this section with some special cases where the global existence of smooth solutions is known:

- Small initial data: Glassey-Strauss [11, 12], Schaeffer [21].

- Perturbations of global solutions: Rein [18].

- Small dimensions: Glassey-Schaeffer $[6,7]$.

Unfortunately, it seems that none of the methods used in those specific cases can be extended to the full three-dimensional problem.

\section{RVM as a non-resonant coupling}

3.1. Kinetic formulation. The main difficulty when dealing with the regularity issues in the RVM system is the hyperbolic nature of the fields equations. It looks as if one derivative were 'lost' when evaluating $E$ and $B$ from the distribution function $f$ in Maxwell's equations. This problem was first addressed by GlasseyStrauss in their paper [8] by using rather tricky computations on the expressions of 
the fields. We describe here a more intrinsic approach used in [2]. A first step is to recast the equations as a coupling between a wave equation and Vlasov equation. Let us introduce a function $u \equiv u(t, x, \xi)$ solving

$$
\square_{t, x} u=f, \quad u_{\mid t=0}=0, \quad \partial_{t} u_{\mid t=0}=0 .
$$

This function should be considered as a microscopic electromagnetic potential. Indeed, let $A^{0} \equiv A(t, x)$ be a vector-valued function verifying

$$
\square_{t, x} A^{0}=0, \quad A_{\mid t=0}^{0}=A^{i n}, \quad \partial_{t} A_{\mid t=0}^{0}=-E^{i n},
$$

where $A^{i n}$ is chosen such that $\nabla_{x} \cdot A^{i n}=0$ and $\nabla_{x} \wedge A^{i n}=B^{i n}$. One is then able to compute the electromagnetic potentials in the Lorentz gauge by

$$
\phi(t, x)=\int u(t, x, \xi) d \xi, \quad A(t, x)=A^{0}(t, x)+\int u(t, x, \xi) v(\xi) d \xi .
$$

The expressions for the fields follow:

$$
\begin{aligned}
& E=-\partial_{t} A^{0}-\int \partial_{t} u v(\xi) d \xi+\int \nabla_{x} u d \xi \\
& B=\nabla_{x} \wedge A^{0}+\int \nabla_{x} \wedge u v(\xi) d \xi
\end{aligned}
$$

As a consequence, the RVM system can be rewritten as

$$
\begin{aligned}
\square_{t, x} u & =f, \\
\partial_{t} f+v(\xi) \cdot \nabla_{x} f & =-(E+v \wedge B) \cdot \nabla_{\xi} f,
\end{aligned}
$$

with fields given by (3.1), (3.2).

3.2. Non-resonant coupling. Using the terminology of $[1,15]$, this coupling is called non-resonant, because $|v(\xi)|<1$ holds for any $\xi \in \mathbb{R}^{3}$. That is, the particles' speeds in the transport operator are strictly less that the propagation speed in the wave operator, i.e. 1. The point is that under this hypothesis, a regularizing phenomena occurs for moments of $u$ against test functions $\chi \equiv \chi(\xi) \in \mathcal{C}_{c}^{\infty}\left(\mathbb{R}^{3}\right)$. But in view of the expressions above, the fields can be written essentially as a linear combination of derivatives of such moments, provided that $f$ - and hence $u$ - is compactly supported in the $\xi$ variable. This is precisely the assumption (2.1) in the statement of theorem 2.1. The next section explains how regularity issues are treated in this framework.

\section{Division lemma}

4.1. Statement of the lemma. Let $Y \in \mathcal{D}^{\prime}\left(\mathbb{R}^{4}\right)$ be the forward fundamental solution of the wave operator:

$$
Y(t, x)=\frac{\mathbf{1}_{t>0}}{4 \pi t} \delta(|x|-t) .
$$

Notice that the distribution $Y$ is homogeneous of degree -2 in $\mathbb{R}^{4}$. Let $\mathcal{M}_{m}$ be the space of $C^{\infty}$ homogeneous functions of degree $m$ on $\mathbb{R}^{4} \backslash 0$. Below, we use the notation

$$
x_{0}:=t, \quad \text { and } \partial_{j}:=\partial_{x_{j}}, \quad j=0, \ldots, 3 .
$$

With each $v \in \mathbb{R}^{3}$ is associated the streaming operator $T=\partial_{t}+v \cdot \nabla_{x}$. The following lemma is the cornerstone of [2].

Lemma 4.1 (Division lemma). For each $v \in \mathbb{R}^{3}$ such that $|v|<1$, 
- there exists functions $a_{i}^{k} \equiv a_{i}^{k}(t, x)$ where $i=0, \ldots, 3$ and $k=0,1$, such that $a_{i}^{k} \in \mathcal{M}_{-k}$ and

$$
\partial_{i} Y=T\left(a_{i}^{0} Y\right)+a_{i}^{1} Y, \quad i=0, \ldots, 3 ;
$$

- there exists functions $b_{i j}^{k} \equiv b_{i j}^{k}(t, x)$ with $i, j=0, \ldots, 3, k=0,1,2$, such that $b_{i j}^{k} \in$ $\mathcal{M}_{-k}$ and

$$
\partial_{i j}^{2} Y=T^{2}\left(b_{i j}^{0} Y\right)+T\left(b_{i j}^{1} Y\right)+b_{i j}^{2} Y, \quad i, j=0, \ldots, 3 ;
$$

- moreover, the functions $b_{i j}^{2}$ satisfy the conditions

$$
\int_{\mathbb{S}^{2}} b_{i j}^{2}(1, y) d \sigma(y)=0, \quad i, j=0, \ldots, 3,
$$

where $d \sigma(y)$ is the rotation invariant surface element on the unit sphere $\mathbb{S}^{2}$ of $\mathbb{R}^{3}$. In both formulas (4.3) and (4.4), $a_{i}^{0} Y, a_{i}^{1} Y, b_{i j}^{0} Y$ and $b_{i j}^{1} Y$ designate, for each $i, j=0, \ldots, 3$, the unique extensions as homogeneous distributions on $\mathbb{R}^{4}$ of those same expressions - which are a priori only defined on $\mathbb{R}^{4} \backslash 0$. Likewise, $b_{i j}^{2} Y$ designates, for $i, j=0, \ldots, 3$ the unique extension as a homogeneous distribution of degree -4 on $\mathbb{R}^{4}$ of that same expressions for which the relation (4.4) holds in the sense of distributions on $\mathbb{R}^{4}$.

4.2. Remarks. The proof is to be found in [2]. Let us give a few comments.

1. The proof is based on the commutation properties of the wave operator with the Lorentz boosts. In particular, the lemma holds almost verbatim in a two-dimensions space. As a result, the approach considered here leads to an equivalent of theorem 2.1 in the two-dimensional case, which required a different proof in [7].

2. Another benefit from this method is to obtain the property (4.5) on coefficients $b_{i j}^{k}$ as a natural consequence when dealing with the homogeneous distributions that appear in the construction.

3. A crucial point in lemma 4.1 is the non-resonant condition $|v|<1$. Of course, in the sequel $v$ will be the relativistic velocity $v(\xi)$ which satisfies the assumption for any $\xi \in \mathbb{R}^{3}$.

4. We claim that the coefficients $a_{i}^{k}$ and $b_{i j}^{k}$ depends smoothly on the vector $v$, see [2]. As a result, for any multi-index $\alpha \in \mathbb{N}^{3}$, we have $\partial_{\xi}^{\alpha} a_{i}^{k} \in \mathcal{M}_{-k}$ and $\partial_{\xi}^{\alpha} b_{i j}^{k} \in \mathcal{M}_{-k}$.

\section{Estimates}

5.1. Estimates on the fields. With lemma 4.1 at our disposal, we are able to derive $L^{\infty}$ estimates for the derivatives of moments of $u$. Assume that $f$ satisfy the assumption (2.1) of theorem 2.1 and let $\theta \in \mathcal{C}_{c}^{\infty}\left(\mathbb{R}^{3}\right)$ be a cut-off function such that $\theta(\xi)=1$ for any $|\xi| \leq r^{*}$ and $\theta(\xi)=0$ when $|\xi|>2 r^{*}$. Then for any $m \in \mathcal{C}^{1}\left(\mathbb{R}^{3}\right)$, we have

$$
\int u(t, x, \xi) m(\xi) d \xi=\int u(t, x, \xi) m(\xi) \theta(\xi) d \xi .
$$

Differentiating this equality, we find for any $i=0, \ldots, 3$,

$$
\int \partial_{i} u(t, x, \xi) m(\xi) d \xi=\int \partial_{i} u(t, x, \xi) m(\xi) \theta(\xi) d \xi .
$$


By definition of $Y$, we have ${ }^{1} u=Y \star\left(f \mathbf{1}_{t>0}\right)$. Using lemma 4.1, it comes

$$
\int u m(\xi) d \xi=\int\left(m \theta a_{i}^{1} Y\right) \star\left(f \mathbf{1}_{t>0}\right) d \xi+\int\left(m \theta a_{i}^{0} Y\right) \star T\left(f \mathbf{1}_{t>0}\right) d \xi,
$$

where the coefficients $a_{i}^{1} \equiv a_{i}^{1}(t, x, \xi)$ and $a_{i}^{0} \equiv a_{i}^{0}(t, x, \xi)$ are given by lemma 4.1 with $v \equiv v(\xi)$. The first term is readily bounded by

$$
\left|\int\left(m \theta a_{i}^{1} Y\right) \star\left(f \mathbf{1}_{t>0}\right) d \xi\right| \leq \frac{4}{3} \pi r^{* 3}\|m\|_{L^{\infty}}\left\|t \theta a_{i}^{1}\right\|_{L^{\infty}} \int_{0}^{t}\|f(s, \cdot, \cdot)\|_{L^{\infty}} d s .
$$

With the a priori estimate (1.11), we infer that it is bounded by a constant. For the second term in (5.1), we use the transport equation (1.6). It comes

$$
T\left(f \mathbf{1}_{t>0}\right)=\nabla_{\xi} \cdot(f(E+v \wedge B)) \mathbf{1}_{t>0}+f^{i n} \delta_{t=0} .
$$

Integrating by parts in the $\xi$ variable,

$$
\begin{aligned}
\int\left(m \theta a_{i}^{0} Y\right) \star T\left(f \mathbf{1}_{t>0}\right) d \xi= & \int \nabla_{\xi}\left(m \theta a_{i}^{0} Y\right) \star\left(f \mathbf{1}_{t>0}(E+v \wedge B)\right) d \xi \\
& +\int\left(m \theta a_{i}^{0} Y\right) \star_{x} f^{i n} d \xi
\end{aligned}
$$

The last term depends only on the initial data and is easily estimated. The first one is bounded above by:

$$
\frac{4}{3} \pi r^{* 3}\|m\|_{W^{1, \infty}}\left\|m \theta a_{i}^{1}\right\|_{L_{t, x}^{\infty} W_{\xi}^{1, \infty}}\|f\|_{L^{\infty}} \int_{0}^{t}\|(E, B)(s, \cdot, \cdot)\|_{L^{\infty}} d s .
$$

Let us define the following quantity

$$
I_{m}(t)=\sup _{i=0, \ldots, 3}\left\|\int \partial_{i} u(t, \cdot, \xi) m(\xi) d \xi\right\|_{L^{\infty}\left(\mathbb{R}^{3}\right)} .
$$

The inequalities above lead to

$$
I_{m}(t) \leq C\left(r^{*}\right)\left(1+\int_{0}^{t}\|(E, B)(s, \cdot)\|_{L^{\infty}} d s\right) .
$$

But we know from (3.1) and (3.2) that $E$ and $B$ are derivatives of moments of $u$ up to a term that depends only on the initial data. Thus, one can apply Gronwall's lemma which shows that for any $m, I_{m}(t) \leq C\left(r^{*}\right)$ and hence

$$
\|(E, B)(t, \cdot)\|_{L^{\infty}} \leq C\left(r^{*}\right) .
$$

5.2. Estimates on the gradient of the fields. The same line can be applied to derive bounds on the second order derivatives of moments of $u$. We shall omit the whole computations and only study the scheme of the proof. As above, the division lemma is used to exchange any arbitrary derivative with the transport operator:

$$
\begin{aligned}
\int \partial_{i j} u m d \xi= & \int\left(m b_{i j}^{2} Y\right) \star\left(f \mathbf{1}_{t>0}\right) d \xi+\int\left(m b_{i j}^{1} Y\right) \star T\left(f \mathbf{1}_{t>0}\right) d \xi \\
& +\int\left(m b_{i j}^{0} Y\right) \star T^{2}\left(f \mathbf{1}_{t>0}\right) d \xi
\end{aligned}
$$

\footnotetext{
${ }^{1} \star$ denotes the convolution in the $(t, x)$ variables, while $\star_{x}$ is the convolution in $x$ only.
} 
The second term is very similar to (5.2) and is managed similarly. It can be bounded by a constant $C\left(r^{*}\right)$. The third one requires an additional treatment, as can be seen by computing

$$
T^{2} f=T \nabla_{\xi} \cdot(f K)=\left[T, \nabla_{\xi}\right](f K)+\nabla_{\xi} \cdot\left(\nabla_{\xi} \cdot(f K) K+f T K\right) .
$$

Since we aim at avoiding differentiating $f$, an additional use of lemma 4.1 is needed to deal with the first order operator $\left[T, \nabla_{\xi} \cdot\right]$. It leads eventually to the following upper bound:

$$
C\left(r^{*}\right)\left(1+\int_{0}^{t}\left\|\left(\nabla_{x} E, \nabla_{x} B\right)(s, \cdot)\right\|_{L^{\infty}} d s\right) .
$$

It remains to consider the more singular term in the decomposition (5.4). The key point here is the property (4.5) satisfied by the coefficients $b_{i j}^{2}$ which allows by a principal value argument to estimate it by

$$
C\left(r^{*}\right)\left(1+\ln \left(1+\left\|\nabla_{x} f\right\|_{L^{\infty}\left([0, t] \times \mathbb{R}^{6}\right)}\right)\right) .
$$

Bringing together the estimates for the three parts, and applying Gronwall's lemma shows that

$$
\left\|\left(\nabla_{x} E, \nabla_{x} B\right)(t, \cdot)\right\|_{L^{\infty}\left(\mathbb{R}^{3}\right)} \leq C\left(r^{*}\right)\left(1+\ln \left(1+\left\|\nabla_{x} f\right\|_{L^{\infty}\left([0, t] \times \mathbb{R}^{6}\right)}\right)\right) .
$$

5.3. Estimates on $f$. Differentiating Vlasov's equation, then integrating in $t$ gives

$$
\left\|\nabla_{x, \xi} f(t, \cdot, \cdot)\right\|_{L^{\infty}} \leq\left\|\nabla_{x, \xi} f^{i n}\right\|_{L^{\infty}}+C \int_{0}^{t} 1+\left\|\left(\nabla_{x} E, \nabla_{x} B\right)(s, \cdot)\right\|_{L^{\infty}} d s .
$$

Using the bound derived earlier, we get

$$
\left\|\nabla_{x, \xi} f\right\|_{L^{\infty}\left([0, t] \times \mathbb{R}^{6}\right)} \leq C\left(r^{*}\right)+C\left(r^{*}\right) \int_{0}^{t} \phi\left(\left\|\nabla_{x, \xi} f\right\|_{L^{\infty}\left([0, s] \times \mathbb{R}^{6}\right)}\right) d s,
$$

with an almost linear growth rate $\phi(t)=(1+t) \ln (1+t)$. Thus the use of Gronwall's lemma is allowed, and we infer a bound on the gradient of $f$. The bound on the gradient of the fields follows, which ends the proof of theorem 2.1.

\section{REFERENCES}

[1] F. Bouchut, F. Golse and C. Pallard, Nonresonant smoothing for coupled wave + transport equations and the Vlasov-Maxwell system, Rev. Mat. Iberoamericana, 20, 865-892, 2004.

[2] F. Bouchut, F. Golse and C. Pallard, Classical solutions and the Glassey-Strauss theorem for the $3 D$ Vlasov-Maxwell system, Arch. Rational Mech. Anal., 170, 1-15, 2003.

[3] F. Bouchut, F. Golse and M. Pulvirenti, Kinetic Equations and Asymptotic Theory, L. Desvillettes \& B. Perthame eds.. Series in Applied Mathematics, 4. Gauthier-Villars, Editions Scientifiques et Médicales Elsevier, Paris; North-Holland, Amsterdam, 2000.

[4] R. DiPerna and P.-L. Lions, Global weak solutions of Vlasov-Maxwell systems, Comm. Pure. Appl. Math., 42, 729-757, 1989.

[5] R. Glassey, The Cauchy Problem in Kinetic Theory, SIAM, 1996.

[6] R. Glassey and J. Schaeffer, The "two and one-half dimensional" relativistic Vlasov Maxwell system, Comm. Math. Phys., 185, 257-284, 1997.

[7] R. Glassey and J. Schaeffer, The relativistic Vlasov-Maxwell system in two space dimensions. I, II, Arch. Rational Mech. Anal., 141, 331-374, 1998. 
[8] R. Glassey and W. Strauss, Singularity formation in a collisionless plasma could occur only at high velocities, Arch. Rational Mech. Anal., 92, 59-90, 1986.

[9] R. Glassey and W. Strauss, Large velocities in the relativistic Vlasov-Maxwell equations. J. Fac. Sci. Univ. Tokyo, Sect IA, Math., 36, 615-627, 1986.

[10] R. Glassey and W. Strauss, High velocity particles in a collisionless plasma, Math. Meth. Appl. Sci., 9, 46-52, 1987.

[11] R. Glassey and W. Strauss, Absence of shocks in an initially dilute collisionless plasma, Comm. Math. Phys., 113, 191-208, 1987.

[12] R. Glassey and W. Strauss, Global existence for the relativistic Vlasov-Maxwell with nearly neutral initial data, Comm. Math. Phys., 119, 353-384, 1998.

[13] E. Horst, On the asymptotic growth of the solutions of the Vlasov-Poisson system, Math. Meth. Appl. Sci., 16, 75-85, 1993.

[14] S. Klainerman and G. Staffilani, A new approach to study the Vlasov-Maxwell system, Comm. Pure Appl. Anal., 1(1), 103-125, 2002.

[15] C. Pallard, Nonresonant smoothing in Sobolev spaces for coupled wave + transport equations, Bull. Sci. Math., 127, 705-718, 2003.

[16] C. Pallard, On the boundedness of the momentum support of solutions to the relativistic VlasovMaxwell system, Indiana Univ. Math. J., 54, 1395-1409, 2005.

[17] K. Pfaffelmoser, Global classical solutions of the Vlasov-Poisson system in three dimensions for general initial data, J. Diff. Eqns., 95, 281-303, 1992.

[18] G. Rein, Generic global solutions of the relativistic Vlasov-Maxwell system of plasma physics, Comm. Math. Phys., 135, 41-78, 1990.

[19] G. Rein, Global weak solutions to the relativistic Vlasov-Maxwell system revisited, Comm. Math. Sci., 2, 145-158, 2004.

[20] J. Schaeffer, Global existence of smooth solutions to the Vlasov-Poisson system in three dimensions, Comm. P. D. E., 16, 1313-1335, 1991.

[21] J. Schaeffer, A small data theorem for collisionless plasma that includes high velocity particles, Indiana Univ. Math. J., 53, 1-34, 2004. 